\title{
Tidal flares and rates from an archival cluster survey
}

\author{
W.P. Maksym ${ }^{1,2,3, a}$ \\ ${ }^{1}$ Northwestern University, CIERA, 2131 Tech Drive, Evanston, IL 60208-2900, USA \\ ${ }^{2}$ Northwestern University, Department of Physics and Astronomy, 2131 Tech Drive, Evanston, IL 60208-2900, \\ USA \\ ${ }^{3}$ University of Alabama, Department of Physics and Astronomy, 206 Gallalee Hall, 514 University Blvd., \\ University of Alabama, Tuscaloosa, AL 35487-0324, USA
}

\begin{abstract}
Tidal disruption flares (TDFs) are potent indicators of the co-evolution of galaxies and the massive black holes (MBHs) which they host in their nuclei, both in terms of the individual black holes revealed by tidal flares and in terms of the overall disruption rate. We examine key concerns regarding the disruption rate from an observational perspective. We also present the findings to date of an archival survey of galaxy clusters using X-ray variability selection and multi-wavelength follow-up. We present a disruption rate that is consistent with other observational and theoretical findings to date, as well as two noteworthy X-ray flares which we attribute to tidal disruption events.
\end{abstract}

\section{THE TDF RATE: OBSERVATIONAL CONSIDERATIONS}

\subsection{Indicators of the MBH Population}

Observations of TDFs are of great interest from the perspective of galactic evolution. In particular there are well-known observational correlations between the mass of a given black hole $\left(M_{\bullet}\right)$ and the dispersion of the surrounding nuclear stellar cluster $\left(M_{\bullet}-\sigma\right)$, and similarly between $M_{0}$ and the luminosity of the host galaxy's bulge $\left(M_{\bullet}-L\right)[1]$.

This relationship becomes challenging to observe at the low end of the MBH population, owing in part to the intrinsic faintness of the host galaxies and the difficulty involved in resolving the MBH radius of gravitational influence at large distances for small $M_{\text {. }}$. But it is of particular importance to hierarchical models of galaxy formation and evolution, as the assumed initial scenarios for $\mathrm{MBH}$ formation may be reflected in the local MBH population today. In particular, different MBH progenitor scenarios such as Population III stars, primordial "seed" black holes, and runaway stellar mergers in the early universe may mean the difference between a flat $\mathrm{MBH}$ distribution today and one that continues the $\left(M_{\bullet}-\sigma\right)$ relation down to much lower masses.

\subsection{Rates from prior observation and theory}

A well-established body of dynamical theory exists to predict the tidal disruption rate $(\gamma)$ via semi-analytical treatments of the loss-cone problem, so measurements of $\gamma$ may also test the validity of different models. For example, some semi-analytical treatments predict $\gamma$ that rises with smaller $M_{\bullet}$, as per [2] and is sensitive to black hole spin effects at larger $M_{\bullet}$ [3]. Numerical treatments large enough to model a nuclear stellar cluster have been rare until recently, however, and provide some indication that the inverse is true [4].

Observational inferences of $\gamma$ are complicated by the fact that numerous selection effects come into play which

a e-mail: wpmaksym@bama.ua.edu may strongly affect the luminosity $L$ and duration of a TDF in a given survey band. There may be strong deviations from the commonly assumed $L \propto t^{-5 / 3}$ evolution (based on the expected accretion rate) depending upon (e.g.) stellar structure, as well as band-specific effects from disk cooling, obscuration, and super-Eddington winds [5-8].

Multiple inferences of the disruption rate using varied selection techniques are therefore useful, as they help reduce the biases of a specific technique or may provide insight into a specific physical process affecting the disruption rate (see Fig. 1 for a graphical overview). Comparison of different rate estimates also has the potential to allow direct comparison of the extent of these selection effects, and thereby an investigation of the relevant physical parameters. Reliable estimates of the rate are difficult due to the Poisson uncertainty inherent in a population sample as small as is typical for prior TDF studies. But TDF studies dependent upon identification of an X-ray, UV or optical flare assumed to be generated by an accretion-disk or other similar scenario seem to be generally consistent with $\gamma \sim$ few $\times 10^{-5}$ galaxy $^{-1}$ year $^{-1}$ [9-12].

The discovery of jetted relativistic flares also present a statistical opportunity to constrain the physics of jet formation via the frequency by which relativistic flares are identified. With 2 relativistic TDFs found over 7 years of the Swift mission, Swift J1644+57 and Swift J2058+05 are consistent with $\gamma \sim$ few $\times 10^{-5}$ galaxy $^{-1}$ year $^{-1}$ for a $\sim 1^{\circ}$ beaming angle, or some combination of wider beaming angle and reduced jet fraction $f_{\text {jet }}$ [13]. Radio surveys may detect relativistic jets without line-of-sight beaming and are therefore more sensitive to the rate of jet formation $\gamma_{j e t}=\gamma \times f_{\text {jet }}$. Archival radio surveys are consistent with $\gamma_{j e t} \lesssim 10^{-6} \mathrm{Mpc}^{-3}$ year $^{-1}$.

\section{AN ARCHIVAL STUDY OF GALAXY CLUSTERS}

With our collaborators, we have undertaken a search for TDFs selected via archival X-ray data from Chandra and XMM-Newton $[15,17,18]$. The $\mathrm{X}$-ray band is an 


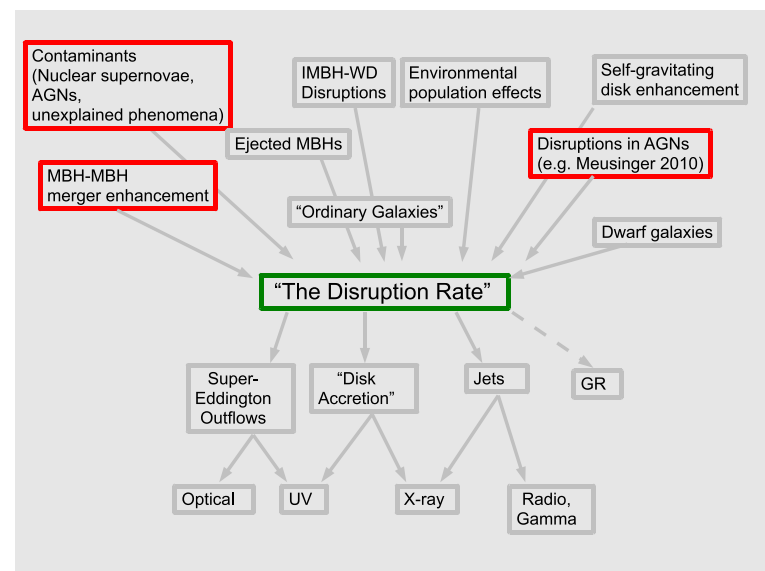

Figure 1. Top: physical scenarios affecting estimates the disruption rate, with potential sources of false positives and false negatives in red. Bottom: TDE-associated phenomena and the regimes by which they may be observed [15, and references therein, as well as other proceedings at this conference].

advantageous choice for finding new TDFs, as it is likely to contain the bulk of the bolometric luminosity and may be the most likely regime in which $t^{-5 / 3}$ decay may be observed over long timescales [8]. TDFs are expected to be quite luminous in $\mathrm{X}$-rays compared to the host emission. The highest energy X-rays are also less likely suffer from host absorption and provide a means of estimating the temperature of the inner accretion disk.

$\mathrm{X}$-ray surveys of rare transients (such as TDFs) are difficult given the limited fields-of-view (FOVs) of focusing X-ray telescopes. But hundreds of galaxy clusters exist in the Chandra and XMM-Newton archives, many of which have been observed multiple times. In a rich cluster, we therefore observe as many as $\sim 1000$ galaxies with a single pointing, maximizing the chance that any given pointing will observe a TDF. Galaxy clusters are also provide the advantages of an easy "first estimate" of the host galaxy distance, prior to any spectroscopic data. Finally, they provide a self-consistent galaxy population by which to calculate $\gamma$, and may help illustrate specific environmental effects relevant to $\gamma$. For example, if most dwarf spheroidal galaxies host MBHs which disrupt stars at elevated rates, the large numbers of these spheroidals in galaxy clusters may dominate the TDF rate [2].

There exist several challenges to this analysis. X-ray mirror point spread functions (PSFs) are typically highly variable across the FOV, making difference imaging (as is common for optical surveys) impossible. Instrumental effective area also varies strongly across instruments, photon energies, within a FOV, or even along a mission timeline. There are also inherent limitations to archival surveys, such as instrumental pointings which are strongly non-ideal (due to lack of overlap) and the inability to promptly follow up source detections to study broadband source evolution. This last issue is particularly critical to distinguishing TDF candidates from TDF impostors. The $\mathrm{X}$-ray sky is inherently variable, and strongly variable $\mathrm{X}$ ray emission may be seen from supernovae, various types of AGNs, and other objects. In light of some of these challenges, we are therefore in the process of automating our source analysis techniques to greater reliability and versatility [16, poster this conference].

\section{A TIDAL FLARE IN A1689 (AND MANY NON-DETECTIONS)}

The first results of this survey revealed one likely TDF, and provided a preliminary estimate of $\gamma$ from a single galaxy cluster (and one of the richest in the Abell catalog), Abell 1689 [17]. Out of 226 detected X-ray sources, one was revealed to be strongly variable $(\times 30)$ and supersoft ( $k T \sim 0.1 \mathrm{keV}$ or $\Gamma \sim 5$ ), consistent with expected traits of non-relativistic TDFs and inconsistent with various classes of variable X-ray objects, including most galactic objects and AGNs. Spectroscopy of the associated host galaxy via the Hobby-Eberly Telescope set strong limits on AGN diagnostic lines such as $\mathrm{H} \alpha, \mathrm{H} \beta$ and [O III], indicating the host galaxy is unlikely to be either a Narrow Line Seyfert 1 (which may be strongly variable in soft X-rays) or a Seyfert 2 galaxy. The only plausible alternative would be an unusual strong flare from a normally weak accretion disk.

Models of the A1689 galaxy population down to $K_{S} \lesssim$ 19.2 suggest $\gamma \sim 1.2 \times 10^{-4}$ galaxy $^{-1}$ year $^{-1}$ for $3 \times 10^{5} \mathrm{M}_{\odot}<$ $M_{\bullet}<3 \times 10^{7} \mathrm{M}_{\odot}$ (with $M_{\bullet} \gtrsim 3 \times 10^{7} \mathrm{M}_{\odot}$ assumed to likely be a small fraction of both the $M_{\bullet}$ and TDF populations) [17]. However, subsequent analysis of additional clusters (bringing the survey total to 10) has failed to detect additional TDF candidates (TDFCs) with variability $\times 5$ or greater that is not also associated with a pre-existing AGN, except in the case of Abell 1795 as explained in $\S 4[15,18]$. The failure to identify plausible TDFCs in these additional clusters forces a downward revision to $\gamma \sim 4.5 \times 10^{-5}$ galaxy $^{-1}$ year $^{-1}$ according to this survey [15].

\section{A REMARKABLE LIKELY TDF IN A1795}

In subsequent analysis of additional galaxy clusters observed multiple times by some combination of Chandra and XMM-Newton, a strong ( $\sim 700$ counts in a single Dec. $1999 \sim 20 \mathrm{ks}$ epoch) X-ray source with negligible counts $\gtrsim 2 \mathrm{keV}$ was identified at a projected distance of $\sim 50 \mathrm{kpc}$ from the centrally located brightest cluster galaxy in multiple Chandra pointings $[15,18]$. The brightest detection was also the first Chandra pointing of A1795, which has since been followed numerous times for a total of 28 Chandra pointings and 1 XMM-Newton pointing. Most of these Chandra pointings are due to A1795 being a Chandra calibration source. As with the A1689 flare, the A1795 source demonstrated characteristics typically expected of TDFs in X-rays: it was extremely variable ( $\times 30$ over $\sim 4$ years in Chandra counts alone), consistent with X-ray luminosity $L_{X}(0.2-2 \mathrm{keV}) \sim 2 \times 10^{42} \mathrm{erg} \mathrm{s}^{-1}$ if it is associated with the cluster $(z \sim 0.062)$, and has a supersoft spectrum consistent with a blackbody of $k T \sim$ $0.08 \mathrm{keV}$, but is better fit by a power law $\Gamma \sim 4.2$ and is associated with faint $(V \sim 22.5)$ extended object WINGS J134849.88+263557.5 (WINGS J1348) [19].

Several characteristics of this TDFC are noteworthy. Firstly, strong X-ray detections of TDFs are rare and 


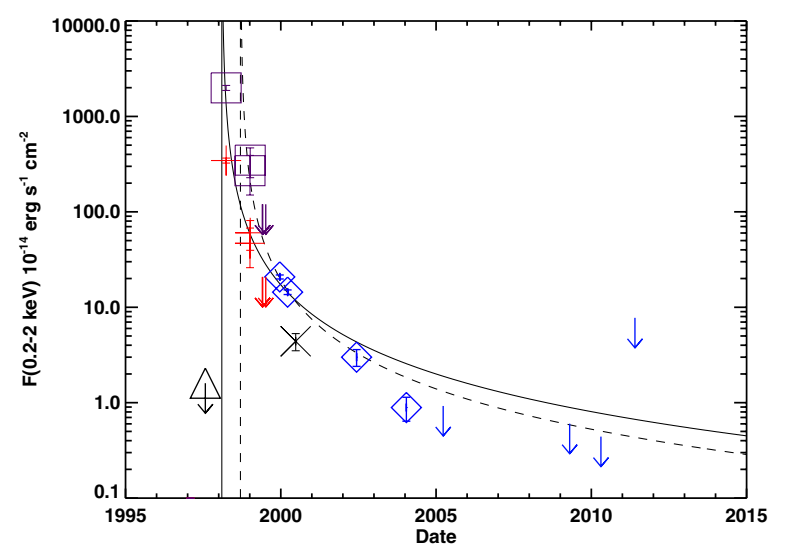

Figure 2. 0.2-2.0 keV light curve of the TDFC in A1795. Solid and dashed lines infer $t^{-5 / 3}$ decay after different disruption times prior to earliest Chandra data. Symbols are: blue diamonds and arrow upper limits (Chandra), black $\times(X M M-N e w t o n)$, black triangle (latest ROSAT upper limit). Different models for EUVE data assume a linear relationship between count rate and the earliest Chandra flux (red crosses) or an absorbed power law of $\Gamma=4.21$, as per the best fit to early Chandra data. $10^{-14} \mathrm{erg} \mathrm{cm}^{-2} \mathrm{~s}^{-1} \sim 10^{41} \mathrm{erg} \mathrm{s}^{-1}$ at $z \sim 0.062$ (A1795) [15, 18].

typically only allow very rough estimates of $L_{X}$ [17] or at best upper limits [11]. A detection of 700 counts makes gives the A1795 flare some of the best counting statistics of any non-relativistic TDFCs identified with the spectral range and resolution of Chandra or XMM-Newton, allowing it to be meaningfully fit to spectral models across multiple epochs [20,21, for comparison].

Secondly, the repeated Chandra calibration observations provide some of the best long-term (over 13 years) temporal coverage of any TDFC (see Fig. 2). All > 2005 epochs provide only upper limits to $L_{X}$, supporting the argument that WINGS J1348 is not a persistent AGN.

Some of the most critical and interesting aspects of this TDFC depend upon the two considerations of whether WINGS J1348 is actually a cluster member and whether WINGS J1348 is actually a flaring AGN. We have obtained Magellan Echellette spectroscopy which has a poor $(\sim 2)$ continuum signal-to-noise ratio, which does not allow an absorption line redshift, but does (due to the faintness of the continuum) place constraints of $F \lesssim 10^{-16} \mathrm{erg} \mathrm{cm}^{-2} \mathrm{~s}^{-1}$ on $\mathrm{H} \alpha, \mathrm{H} \beta$ and [O III] (as for the A1689 flare).

The color $(B-V=0.8)$ disfavors a Seyfert 1 of any sort, but we must consider the possibility of a flaring Seyfert 2, as Seyfert 2 galaxies may be difficult to distinguish from inactive galaxies and are one of the more common sources of supersoft X-ray variability that is expected from TDFs [22]. The putative host galaxy is sufficiently faint that despite numerous observations of A1795 between FUV (GALEX) and FIR Herschel, WINGS $\mathrm{J} 1348$ is only detected in $B, V$. A comparison of these broadband limits to those of Seyfert 2 galaxies in SWIRE suggests that WINGS J1348 is fainter at $1 \mu \mathrm{m}-200 \mu \mathrm{m}$ than typical Seyfert 2 galaxies, although that explanation cannot be ruled out entirely. Deeper spectroscopy is necessary to conclusively eliminate a Seyfert 2 explanation and confirm cluster membership (which is consistent with color-magnitude diagrams of A1795).

Confirmation of cluster membership is critical to confirming one of the most interesting implications of the flare in WINGS J1348. WINGS photometry and an HST WFPC2 spatial profile imply that WINGS J1348 must be a $r \lesssim 300$ pc dwarf galaxy if it is a member of A1795. The faintness and extent of the galaxy therefore imply WINGS J1348 would be an order of magnitude less massive than POX 52 or Henize 2-10, two of the least massive galaxies known to host MBHs [24, 25].

From the earliest Chandra data, the Eddington limit implies $M_{\bullet} \gtrsim 2 \times 10^{4} \mathrm{M}_{\odot}$, and a blackbody disk model is consistent with $\mathrm{M}_{\odot} \sim 5 \times 10^{5} \mathrm{M}_{\odot}$. However, earlier observations of A1795 with the EUVE DS camera display an EUV/supersoft X-ray transient as early as March 1998, most likely associated with the WINGS J1348 X-ray flare. In this earliest flare epoch, the $\sim 2350$ net source counts are $\gtrsim 2 \times$ brighter than the central $2^{\prime} .7$ of the cluster core. This flare was first reported as a point source contaminating EUV observations of the A1795 intracluster medium (ICM) [26] and is still visible in later 1998-1999 observations, but with sufficiently poor spatial and spectral resolution that it is difficult to obtain reliable flux estimates against the diffuse ICM emission at these later times.

If this EUV emission is associated with the WINGS J1348 flare, then it appears to be the first reported detection of a TDF at photon energies lower than XMM-Newton $(k T \lesssim 0.1 \mathrm{keV})$, which is of interest to both constraining the bolometric luminosity of TDFs at those energies, as well as possibly examining the EUV absorption forest predicted by [6] via the spectroscopic instruments onboard EUVE.

The EUV emission also implies a much larger $M_{\bullet}$ via a larger (albeit very uncertain) Eddington luminosity at early times and possibly as much as a $\times 30$ bolometric correction to X-ray observations. $M_{\bullet}$ may be $\gtrsim 10^{6} \mathrm{M}_{\odot}$. As with Henize 2-10, this is very difficult to reconcile with models of hierarchical galaxy formation [25]. However, given the close proximity to the cluster core $(\gtrsim 50 \mathrm{kpc}$ ), WINGS J1348 may be the core remnant of a larger galaxy that has experienced tidal stripping during its passage through the cluster core.

Also of note is that while the XMM-Newton pipeline processing failed to detect the flare against the ICM, the source was detectable by wavelet software when the event file was filtered to $0.1 \mathrm{keV}<k T<0.5 \mathrm{keV}$ and rebinned to finer resolution than the pipeline uses. The success of this technique at identifying a heavily decayed TDFC even in the brightest region of ICM diffuse emission is encouraging for the prospects of $e$ ROSITA to identify TDFs in cluster cores, despite its large $\left(15^{\prime \prime}-30^{\prime \prime}\right)$ PSF.

\section{POPULATION STUDIES OF TDFS}

Although TDFs are rare, there are now enough TDFCs in the literature to attempt to study them as a population [11]. In principle, they can also be used to examine the $M_{\bullet}-L$ relation by alternate means. Inferring $M_{\bullet}$ from the diverse measurements used in the literature is an arduous task, and 


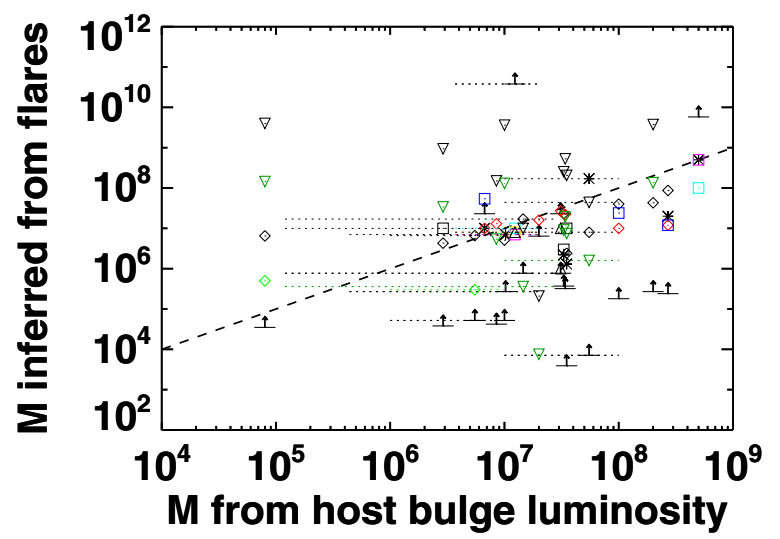

Figure 3. A comparison of TDF-derived values of $M_{\text {. with }}$ $M_{\bullet}-L$ [15, full details therein]. Horizontal bars with arrows represent lower limits due to Eddington luminosity. Diamonds are derived from various classes of thermal models. Squares indicate various classes of light curve decay models. Inverted triangles are inferred from $t^{-5 / 3}$ decay (black, $k=1$; green, $k=3$ ). Horizontal error bars indicate uncertainty in the bulge fraction of the galaxy. Otherwise, errors are comparable to the intrinsic error in $M_{\bullet}-L$. The dashed line indicates $\mathrm{y}=\mathrm{x}$.

uniform surveys will ultimately be better suited to this kind of analysis. But already we can begin to infer certain trends from candidates in the literature [15]. If we assume bandappropriate $M_{\bullet}-L$ for published photometric data of the host galaxies, we can compare against the Eddington limit, broad classes of thermal models, and the light curve time scale (which is to first order a function of the Keplerian orbit of the most-bound debris, and hence $M_{\bullet}$ ).

In Fig. 3, we see a summary of this analysis [15]. Lower limits on $M_{\bullet}$ derived from the Eddington luminosity (black arrows) are broadly consistent with $M_{\bullet}-L$, with the notable exception of Swift J1644+57, for which beaming is not considered. Thermal models, including the assumption that the $L$ originates from a blackbody either near the Schwarzschild radius (X-rays) or the tidal disruption radius (UV, optical, infrared), track $M_{\bullet}-L$ closely. Upper limits from the time of peak observed emission $\left(t_{0}\right)$ and inferred disruption time $\left(t_{D}\right)$ such that $\left(t_{0}-t_{D}\right) \propto k^{-3 / 2}\left(M_{\bullet} / 10^{6} \mathrm{M}_{\odot}\right)^{1 / 2} \beta^{3}(1+z)$ are consistent with a spin-up parameter of $k=1$ and assumed penetration factor $\beta=1$, but are too low for a maximum of $k=3$, although a spin-up correction may not be an appropriate consideration in this analysis [27].

\section{SUMMARY}

Galaxy clusters provide an excellent means of studying tidal flares and the rate at which they occur. By providing an efficient method of TDFC selection via soft $\mathrm{X}$ rays, they provide an important complement to other studies at optical, ultraviolet, infrared, gamma/hard Xrays, and radio surveys. The two examples identified in A1689 and A1795 are $10 \%$ of reported TDFCs to date (and may also be considered in conjunction with a serendipitous TDFC also found in X-ray observations of A3571 via another survey [28]. The Abell 1795 flare presents a particularly striking example of a TDFC due to its likely very unusual host galaxy, its excellent source statistics and time sampling, and due to its detection with a dedicated EUV instrument. The inferred TDF rate of $\gamma \sim$ few $\times 10^{-5}$ galaxy $^{-1}$ year $^{-1}$ is consistent with what appears to be a growing observational consensus, but additional observations are necessary both to identify new examples and to better constrain $\gamma$. The limitations of Poisson statistics from small numbers of TDFCs are particularly severe and significant relative to uncertainties in source populations and flare visibility. The problem of distinguishing between luminous AGN flares and TDFs is a particular difficulty in determining $\gamma$, and the only apparent solutions appear to be greater access to faintsource spectroscopy in the context of TDFC follow-up, or development of broad-band photometric methods that can easily distinguish between these superficially similar phenomena.

We hope to find as many as $\sim 20$ new TDFCs in existing Chandra and XMM-Newton observations of galaxy clusters. We also suggest that X-ray astronomers observing the galaxy cluster ICM investigate or make available the point source populations of their targets in order to better facilitate rapid follow-up of TDFCs. Finally, we suggest that an ROSITA pointed cluster survey has strong potential to identify many new TDFs, given the superiority of its FOV and effective area, relative to Chandra and XMM-Newton.

\section{References}

[1] Gültekin et al., ApJ, 698, (2009) 198

[2] Wang \& Merritt, ApJ, 600, (2004) 149

[3] Kesden, Phys. Rev. D., 85, (2012) 024037

[4] Brockamp et al., MNRAS, 418, (2011) 1308

[5] Strubbe \& Quataert, MNRAS, 400, (2009) 2070

[6] Strubbe \& Quatert, MNRAS, 415, (2011) 168

[7] Lodato, King \& Pringle, MNRAS, 392, (2009) 332

[8] Lodato \& Rossi, MNRAS, 410, (2011) 359

[9] Donley et al., AJ, 124, (2002) 1308

[10] Luo et al., ApJ, 674, (2008) 122

[11] Gezari et al., ApJ, 698, (2009) 1367

[12] Van Velzen et al., ApJ, 741, (2011) 73

[13] Cenko et al., ApJ, 753, (2012) 77

[14] Meusinger et al., A\&A, 512, (2010) A1

[15] Maksym, An X-ray Survey for Tidal Disruption Flares in Rich Clusters of Galaxies (Northwestern University, 2012), Ph.D. Thesis

[16] Wampler-Doty, Maksym \& Ulmer, Survey of Chandra and XMM Galaxy Cluster Archival Data In Search of Tidal Flare Events, (European Space Agency Centre, Tidal Disruption Events and AGN Outbursts Workshop), Poster

[17] Maksym, Ulmer \& Eracleous, ApJ, 722, (2010) 1035

[18] Maksym, Ulmer, Eracleous, Guennou, Ho \& Klein, in preparation, $(2012)$

[19] Varela et al., A\&A, 497, (2009) 667 
[20] Lin et al., ApJ, 738, (2011) 52

[21] Saxton et al., A\&A, 541, (2012) A106

[22] Saxton et al., in Narrow Line Seyfert 1 Galaxies and their Place in the Universe (2011)

[23] Polletta et al., ApJ, 663, (2007) 81
[24] Thornton et al., ApJ, 686, (2008) 892

[25] Reines et al., Nature, 479, (2011) 66

[26] Bowyer, Berghöfer \& Korpela, ApJ, 526, (1999) 592

[27] Gezari, personal communication

[28] Cappelluti et al., A\&A, 495, (2009) L9 